\title{
Small Volume Ionization Chambers Angular Dependence and Its Influence on Point-Dose Measurements
}

\author{
Jon Feldman ${ }^{1,2}$, Itzhak Orion ${ }^{1}$ \\ ${ }^{1}$ Nuclear Engineering, Ben Gurion University of the Negev, Beer Sheva, Israel \\ ${ }^{2}$ Sharett Institute of Oncology, Hadassah University Hospital, Jerusalem, Israel \\ Email: iorion@bgu.ac.il
}

Received 26 November 2015; accepted 1 February 2016; published 4 February 2016

Copyright (C) 2016 by authors and Scientific Research Publishing Inc.

This work is licensed under the Creative Commons Attribution International License (CC BY). http://creativecommons.org/licenses/by/4.0/

(c) (†) Open Access

\begin{abstract}
Purpose: Cylindrical ionization chambers as the PinPoint and the CC01 have high-spatial resolution and cylindrical symmetry in relevance to small fields. Dosimetry of newly advanced technologies that include non-coplanar beams, such as in Gamma-Knife, VMAT and IMRT treatment plans, the angular dependence of the ion chamber is essential. Therefore in this study we simulated two common used ion chambers using a Monte Carlo method. Methods: The angular dependence for the CCO1 and the PinPoint were calculated for a vast angular range in a cylindrical water phantom for two LINAC photon beams, $6 \mathrm{MV}$ and $15 \mathrm{MV}$. The calculations were performed for fully structural description of each ion chamber using Monte Carlo code, in order to reveal unsymmetrical response dose higher than $2 \%$ in medical applications. The calculated dose response, for the CC01 ion chamber, was validated with measurements. Results: The CC01 ion chamber showed a very small angular dependence compared to the PinPoint ion chamber. The relative angular dependence amplitude of the PinPoint was about $12 \%$ in the range of $0^{\circ}-110^{\circ}$. Conclusions: The differences in the ion chambers structure led to the variations in the relative angular dependence amplitude. Therefore, when choosing a detector, not only the volume of the ionization chamber but also its structure must be taken into consideration.
\end{abstract}

\section{Keywords}

Dosimetry, CC01, PinPoint, Linac, Monte Carlo

\section{Introduction}

The complex 3D shapes of new and sophisticated treatment methods, such as Intensity-Modulated Radiation

How to cite this paper: Feldman, J. and Orion, I. (2016) Small Volume Ionization Chambers Angular Dependence and Its Influence on Point-Dose Measurements. International Journal of Medical Physics, Clinical Engineering and Radiation Oncology, 5, 26-32. http://dx.doi.org/10.4236/ijmpcero.2016.51003 
Therapy (IMRT), Volumetric Modulated Arc Therapy (VMAT), Gamma-Knife and Tomo-Therapy treatments often demand that dissymmetry measurement techniques be reviewed. Understanding the limitations when using dosimeters is critical to administer safe implementation. For point-dose measurements of total delivered dose, small volume cylindrical ionization chambers are generally used, due to their excellent stability and linear response to absorbed dose. These cylindrical ionization chambers have high-spatial resolution and cylindrical symmetry in relevance to small fields. In measurements of treatments such as IMRT or VMAT treatment plans, that include non-coplanar beams, the angular dependence of the ion chamber is essential. Limited data is currently available about oblique-incidence response of small cylindrical ionization chambers to megavoltage beams [1] and Gamma-Knife dosimetry [2] [3].

Several studies of ion chamber angular response dependence were recently published. A few publications reported correction factors for the angular dependence of several ion chambers in various energies using a radioactive source, such as ${ }^{137} \mathrm{Cs}$ and ${ }^{60} \mathrm{Co}$.

In the papers of Takata et al. [4] [5] and Kurosawa et al. [6] dose response measurements results versus angle were presented for both small and large ion chambers. In these papers, the presented measured angular dependence was above $3 \%$, and was flattened toward $\pm 0.5 \%$ using a wall correction function by linear extrapolation techniques. The correction is calculated by the elimination of the wall attenuation, the center of electron production factor and the scattering correction function. Apparently, for several ion chambers the angular dependence can be significant.

Araki et al. and Hrshak et al. showed the angular dependence when using different types of detectors (glass rod dosimeter, a diode detector and the PinPoint PTW31006 ionization chamber) to measure in the GammaKnife helmet output factors [2] [3]. Hrshak's study showed an angular dependence of over $10 \%$ for a $4 \mathrm{~mm}$ collimated beam and up to $3 \%$ for bigger beams $(18,14$ and $8 \mathrm{~mm})$. The angular dependence in Hrshak's study was limited to angles in the range of $54^{\circ}-84^{\circ}$.

Two cylindrical chambers, commonly used for radiotherapy dosimetry, PinPoint (PTW) such as used in Hrshak's study, and the CC01 (IBA), were examined in this study in oblique-incidence for low and high energy beams (6 MV and $15 \mathrm{MV}$ ). The angular dependence was first measured for the CC01 chamber for the relevant couch angular range $\left(0^{\circ}-110^{\circ}\right)$. Monte Carlo calculations were carried out for each chamber, and were compared to the measured results for the CC01 and to Hrshak's study for the PinPoint [2].

\section{Materials and Methods}

\subsection{Experimental Setup}

A cylindrical water phantom perpendicular to the beam, dimensions: $15 \mathrm{~cm}$ diameter and $35 \mathrm{~cm}$ height, was designed and set up on the couch in the isocenter of a Varian, Clinac Trilogy, linear accelerator. The IBA Compact Chamber CC01 cylindrical ionization chamber was inserted into the center of the phantom in parallel to the beam plane. This phantom and chamber were positioned using room lasers and the accelerator crosser as can be seen in Figure 1. A comparison between technical data for these chambers can be seen in Table 1. For the IBA Compact Chamber CC01 we used a ruler in order to position the isocenter at the reference measuring point, 2.3 $\mathrm{mm}$ from the chamber end. Portal images from the On Board Imager (OBI) were used to ensure positioning of the phantom and the ion chamber-Figure 2. The ion chamber was connected to a PTW Electrometer UNIDOSE-E Universal Dosimeter 10009. A square $3 \times 3 \mathrm{~cm}^{2}$ field size was set using the LINAC jaws. Measurements were performed for oblique incidence every 10 degrees between $0^{\circ}$ to $110^{\circ}$. Each measurement was obtained for 100 monitor units (MU), and was repeated three times. All measurements were taken both for the 6 MV and $15 \mathrm{MV}$ photon beams.

\subsection{Monte Carlo Simulations}

The Monte Carlo simulations were written using the EGS5 code system [7]. The EGS5 Monte Carlo code system is a new generation of the EGS4 well validated code for photons and electrons transport. The EGS (Electron-Gamma Shower) code system is a general purpose package for the Monte Carlo simulation of the coupled transport of electrons and photons in an arbitrary geometry for particles with energies ranging from above a few $\mathrm{keV}$ up to several hundred $\mathrm{GeV}$.

The EGS5 is a FORTRAN open source program. Since the 1990s, when the previous EGS4 code was released, 


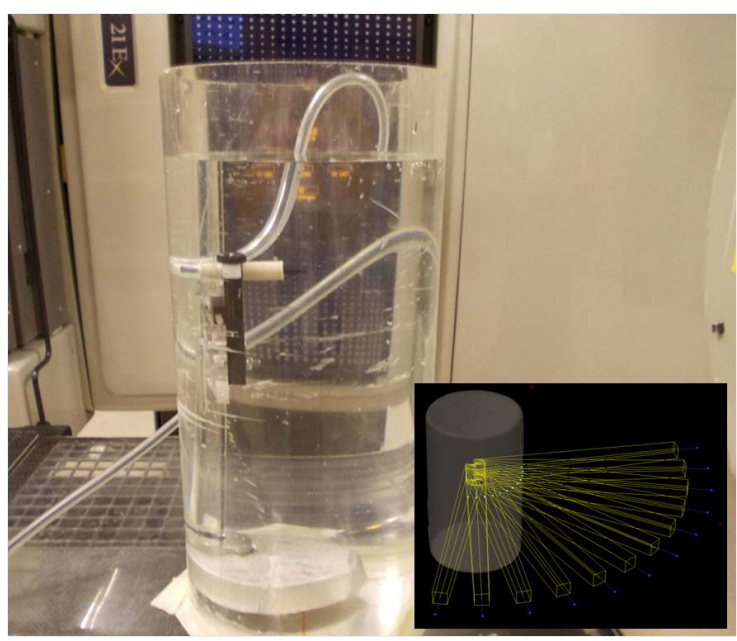

Figure 1. The setup of the CC01 ion chamber in the water phantom. Measurements were taken for every $10^{\circ}$ between angles $0^{\circ}$ to $110^{\circ}$.
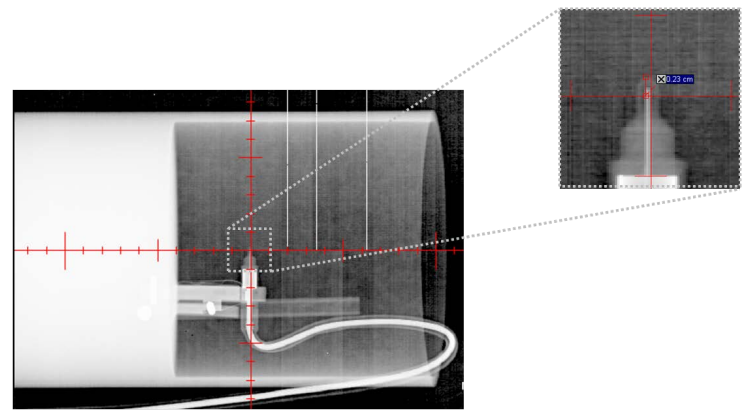

Figure 2. Kilo-voltage portal image of CC01 ion chamber positioned in the water phantom at the isocenter.

Table 1. Technical data for the two ion chambers used in this work the IBA Compact Chamber CC01 and the PTW PinPoint chamber.

\begin{tabular}{ccc}
\hline MODEL & CC01 & PinPoint T31006 \\
VENDOR & IBA DOSIMETRY & PTW \\
DESCRIPTION & CYLINDRICAL & CYLINDRICAL \\
APPROX VOL. [cc] & 0.010 & 0.015 \\
EFF. LENGTH [mm] & 3.6 & 5.0 \\
EFF. DIAMETER [mm] & 2.0 & 2.0 \\
RESPONSE [nC/Gy] & 0.33 & 0.40 \\
LEAKAGE $[10-15$ A] & 0.75 & 4.00 \\
CENTRAL ELECTRODE & STEEL & STEEL \\
WALL MATERIAL & SHONKA C552 & ACRYLIC GRAPHITE
\end{tabular}

it has been used in a wide variety of applications, particularly in medical physics, radiation measurement studies, and industrial development. The EGS5 code system contains, among many other subprograms, four user-called subroutines: BLOCK SET, PEGS5, HATCH, and SHOWER. These routines call other subroutines in the EGS5 code, some of which call two user-written subroutines, HOWFAR and AUSGAB, which respectively define 
geometry, and scoring output. The EGS5 transport code for electrons is fundamentally different and advanced from the previous EGS4 code. The electron step in EGS5 is treated by splitting each step into two segments, and a scattering hinge is applied in between the segments [8].

The user communicates with EGS5 by means of the subroutines mentioned above, which enable him to access variables contained in various COMMON blocks. To use EGS5, the user must write a MAIN program and the subroutines HOWFAR and AUSGAB. The EGS5 consists of newly developed pseudo-random generator, accurate low-energy photon fluorescence transitions and a new algorithm for Bremsstrahlung radiation, which assure reliable results for radiation simulations studies. All used cross-sections for the interactions are described in the EGS5 user manual Appendix E [7]. The EGS5 includes several tools, such as the CG-VIEW used for geometry editing and as a viewer, and the PEGS5 editor for media cross sections definitions. In recent years the use of EGS5 for dosimetry calculations in the field of medical physics has been proven as successful as showed in several papers (e.g. in ref. [9]-[13]).

All kinetic energy cut-offs were set to be at $1 \mathrm{keV}$ throughout this study. The detailed geometry of the same setup, as described above in the experiment, including the IBA Compact Chamber CC01 and the PTW PinPoint, were constructed in detail for the simulations using the CG-VIEW program. The CG-VIEW program is a complementary part of the EGS5 package setup. The media used in the CC01 simulations are: Shonka (C-552) used for outer electrode, steel used for the inner electrode, PEEK used for the chamber stem (based on the compact chamber User's Manual [14]) and air in the active volume. The PinPoint materials are: Steel used for the inner electrode, carbon coating for the inner wall of the outer electrode, Perspex as the wall material, Kapton was used for the inner isolation and air in the active volume. In both cases Perspex was used for the phantom walls, water, and air. Detector dimensions for both chambers are shown in Figure 3(a), Figure 3(b).

The spectra for the $6 \mathrm{MV}$ and the $15 \mathrm{MV}$ beams were taken from the Varian ECLIPSE treatment planning system (ECLIPSETM, Varian Medical Systems). The source definition is an important part of the main code. It has to be carefully mathematically described due to the source position and the rays' direction.

In this work we chose to simplify the source description as a homogeneous, uni-directional photon beam, where the angle of the beam changes according to the "bed angle" (see Figure 1) from the experimental setup. Therefore, there is no necessity to use the BEAMnrc code [15], since we took into consideration in the simulations, only the outgoing beam from the exit of the LINAC head.

The source position vector as depended on the "bed angle" was described as:

$$
\begin{aligned}
& x=66.8 \cdot \cos (\beta)+1.5 \cdot\left(1-2 \varphi_{1}\right) \sin \beta \\
& x=66.8 \cdot \cos (\beta)+1.5 \cdot\left(1-2 \varphi_{1}\right) \sin \beta \\
& z=1.5 \cdot\left(1-2 \varphi_{2}\right)
\end{aligned}
$$

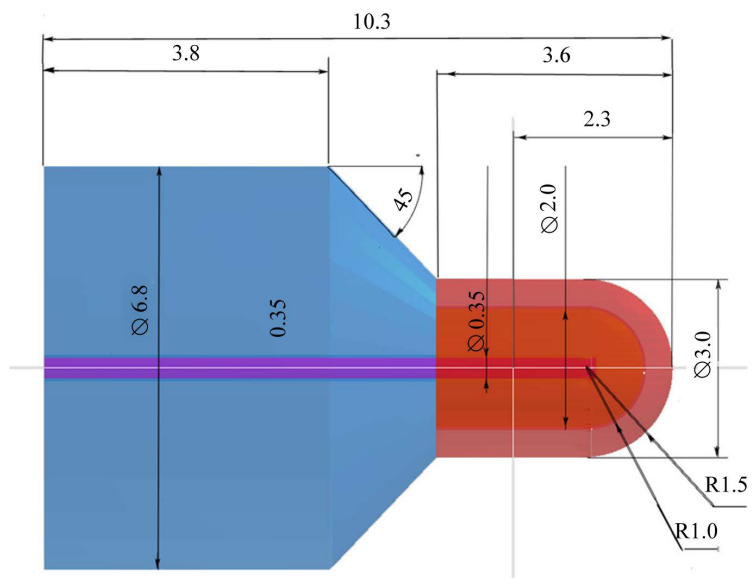

(a)

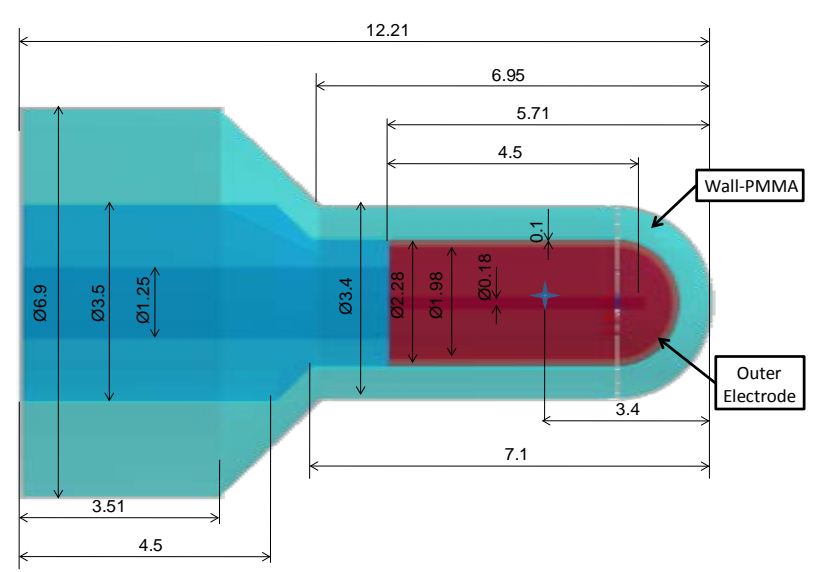

(b)

Figure 3. (a) The CC01 ion chamber geometry and dimensions for the Monte Carlo simulation; (b) The PinPiont ion chamber geometry and dimensions for the Monte Carlo simulation. 
where 66.8 is the approximate distance from the jaw to the iso-center; 1.5 is due to the field opening of $3 \times 3$ $\mathrm{cm}^{2} ; \beta$ describes the "bed angle" and $\varphi_{1}$ and $\varphi_{2}$ are uniform random numbers that were changed for each history.

The source direction vector as depended on the "bed angle" $\beta$ was described as:

$$
\begin{aligned}
& u=-\cos \beta \\
& v=-\sin \beta \\
& w=0.0
\end{aligned}
$$

The spectral data for the simulations introduced to the user code were taken from the ECLIPSE planning system and were fitted to produce cumulative density functions (CDF's). The CDF for each beam energy was obtained using non-linear fit with a uniform random number $\xi$ :

CDF 6 MV:

$$
E[\mathrm{MeV}]=2.08-13.57 \xi+56.70 \xi^{2}
$$

With a coefficient of determination of $R^{2}=0.9581$.

In this spectrum, three discontinuity points were populated using discrete sampling along with the continuous CDF [E from Equation (3)—39\%; $E=0.3 \mathrm{MeV}-20 \% ; E=0.525 \mathrm{MeV}-11 \% ; E=0.825 \mathrm{MeV}-20 \%$ ].

CDF 15 MV:

$$
E[\mathrm{MeV}]=0.346+0.331 \xi-0.064 \xi^{2}+0.005 \xi^{3}-1.534 \times 10^{-4} \xi^{4}
$$

With a coefficient of determination of $R^{2}=0.9677$.

All the simulations were run for 100 million histories (runtime $=62$ hours), and the results were normalized to the results at angle $90^{\circ}$.

\section{Results}

\subsection{Experiment}

The measured angular dependence for the CC01 ion chamber is presented as dependence on the beam energy in Figure 4 symbol with circles (in red) for $6 \mathrm{MV}$ beam and for $15 \mathrm{MV}$ beam. Each measurement was repeated three times, and the average is presented with an estimated error of not more than $0.5 \%$. The IBA Compact Chamber CC01 demonstrated a significant angular dependence, up to $2.4 \%$, the angular dependence was more pronounced for the $6 \mathrm{MV}$ beam than for the $15 \mathrm{MV}$ beam.

\subsection{EGS5 Simulations}

A comparison of the angular dependence between the experimental setup and the EGS5 simulations are presented for the CC01 chamber in both energies in Figure 4 between $0^{\circ}$ to $110^{\circ}$. The EGS5 demonstrated similar angular dependence along the range, up to $3.2 \%$ for the $6 \mathrm{MV}$ and $3.0 \%$ for the $15 \mathrm{MV}$.

For the PinPoint chamber we performed EGS5 simulations for $6 \mathrm{MV}$ and $15 \mathrm{MV}$ energies, as presented in Figure 5. The PinPoint angular response from the simulations showed comparable results to those published by Hrsak et al for the $6 \mathrm{MV}$ collimation of $2 \times 2 \mathrm{~cm}^{2}$ [2]. Hrsak et al. reported the angular dependence for the range of $54^{\circ}-84^{\circ}$ only, and showed a similar trend to the Monte Carlo results in this study. The Monte Carlo results for the PinPoint showed a higher angular dependence compared to the CC01. For the PinPoint chamber in 6 MV beam, we found a difference of up to $11 \%$ and for the $15 \mathrm{MV}$ beam, we found a difference between $(-2) \%$ at $110^{\circ}$ to $12 \%$ at $0^{\circ}$.

\subsection{Simulation Statistical Uncertainties}

In order to estimate the statistical uncertainties in the Monte Carlo simulations results, we set a separate simplified configuration that consisted of the water phantom with a symmetric sphere positioned at the system's isocenter. The sphere was set to be in a volume equal to the CC01 chamber volume, which is the smaller of the two detectors. The aim of this simplified simulation was to study the influence of the statistical fluctuations for each response along the angular range.

In the case of $6 \mathrm{MV}$ with 100 million histories, the fluctuations indicated amplitude up to $2 \%$, as evinced in 


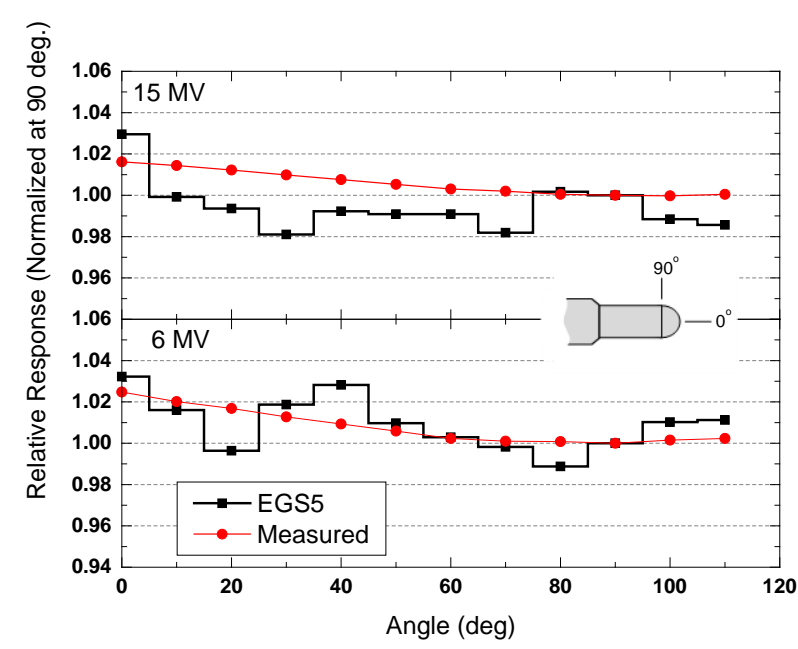

Figure 4. The CC01 ion chamber response vs. angular position, measured compared to the EGS5 simulations, at $6 \mathrm{MV}$ and 15 $\mathrm{MV}$. All results are normalized to the response at $90^{\circ}$.

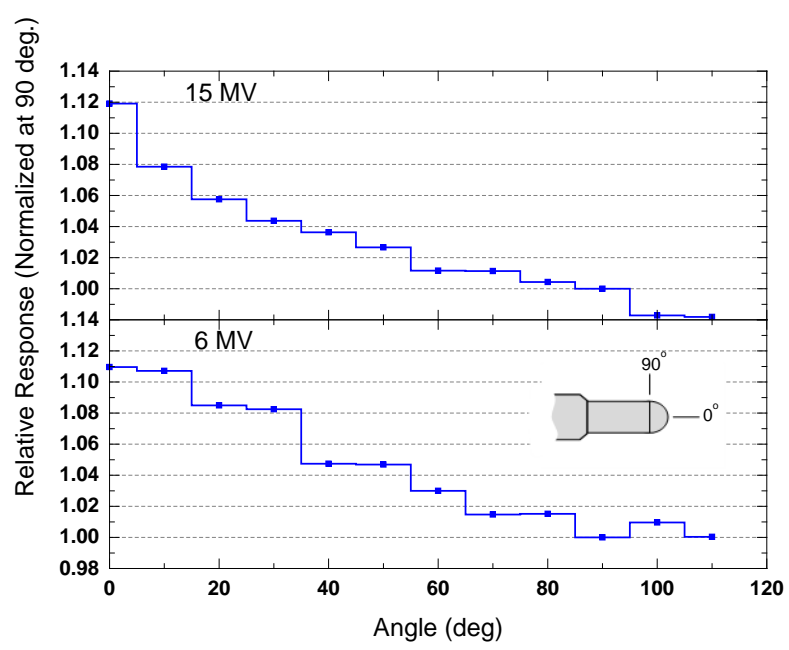

Figure 5. The PinPoint ion chamber calculated response vs. angular position using EGS5 simulations at $6 \mathrm{MV}$ and $15 \mathrm{MV}$. All results are normalized to the response at $90^{\circ}$.

Figure 6. In $15 \mathrm{MV}$ the symmetrical simulation indicated no fluctuations in all directions for 100 million histories (same value for an 8 digits precision). To ensure the statistical significance when using Monte Carlo simulations, we found that it is efficient to perform simplified simulations in order to investigate simulation statistical uncertainties.

\section{Conclusion}

When using different ion chambers, in measurements that include non-coplanar beams, in radiotherapy, the angular dependence of the ion chamber is significant. We found relatively major differences between the two ion chambers in this study. The CC01 ion chamber showed a very small angular dependence compared to the PinPoint ion chamber. This can result from a combination of two reasons. The first is due to the differences in the ion chambers' structures and materials. As showed in Figure 2, the PinPoint chamber consists of an outer wall made of PMMA with an inner coating of graphite used as the outer electrode, whereas in the CC01 the outer electrode is the Shonka (C-552) with no extra outer wall. Kurosawa et al. showed the influence of the wall thickness on the angular dependence [5]. This can explain the stronger angular dependence in the case of the PinPoint. 


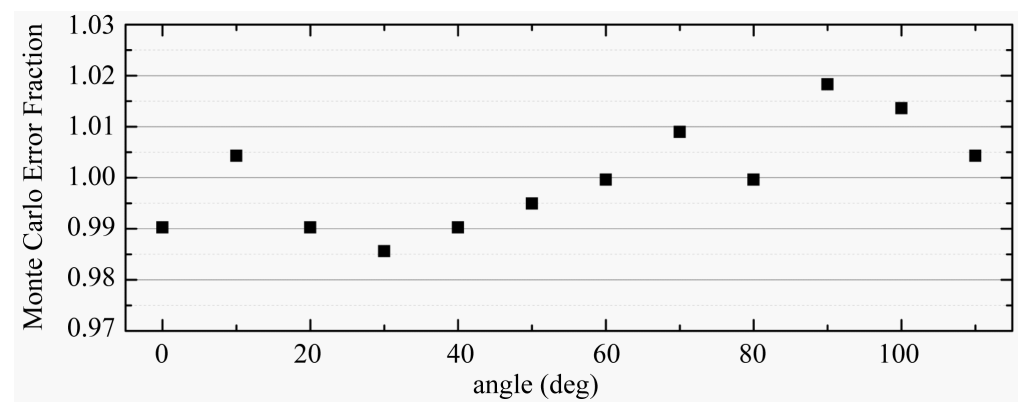

Figure 6. Fluctuations from the EGS5 simplified system simulations vs. beam angle for $6 \mathrm{MV}$ with 100 million histories.

The second reason is the difference in the chambers' length. The PinPoint's active volume is a little longer than the CC01 active volume. Therefore, choosing the suitable ion-chamber when measuring non-coplanar beams is essential. The results in this study showed a clear difference in angular dependence between two ion chambers, although only a field of $3 \times 3 \mathrm{~cm}^{2}$ was used. Extra study could be carried out on angular dependence of these chambers or others in smaller field sizes.

\section{References}

[1] Low, D.A., Moran, J.M., Dempsey, J.F., Dong, L. and Oldham, M. (2011) Dosimetry Tools and Techniques for IMRT. Medical Physics, 38, 1313-1338. http://dx.doi.org/10.1118/1.3514120

[2] Hrsak, H., Majer, M., Grego, T., Bibic, J. and Heinrich, Z. (2014) Correction of Measured Gamma-Knife Output Factors for Angular Dependence of Diode Detectores and PinPiont Ionization Chamber. Physica Medica, 30, 914-919. http://dx.doi.org/10.1016/j.ejmp.2014.09.002

[3] Araki, F., Ikegami, T., Ishidoya, T. and Kubo, H.D. (2003) Measurements of Gamma-Knife Helmet Output Factors Using a Radiophotoluminascent Glass Rod Dosimeter and a Diode Detector. Medical Physics, 30, 1976-1981. http://dx.doi.org/10.1118/1.1587451

[4] Takata, N., Kurosawa, T. and Koyama, Y. (2003) Ionization Chamber Wall Correction Factors and Angular Dependences of Responses in Measuring Air Kerma for Gamma Rays. BIPM Report CCRI(I)/03-18, 4 p.

[5] Takata, N., Kurosawa, T. and Tran, N.T. (2003) Angle Dependence of Signal Currents from Cylindrical Ionisation Chambers. Radiation Protection Dosimetry, 107, 293-296. http://dx.doi.org/10.1093/oxfordjournals.rpd.a006404

[6] Kurosawa, T., Nakata, N. and Koyama, Y. (2003) Angular Dependence of Wall Correction for Cylindrical Cavity Chamber. Proceedings of the Eleventh EGS4 Users’ Meeting in Japan, KEK Proceedings 2003-15, 27-32.

[7] Hirayama, H., Namito, Y., Bielajew, A.F., Wilderman, S.J. and Nelson, W.R. (2006) The EGS5 Code System. SLAC$R-730 /$ KEK Report 2005-8.

[8] Bielajew, A.F. and Wilderman, S.J. (2000) Innovative Electron Transport Methods in EGS5. Proceedings of the 2nd International Workshop on EGS, KEK, Japan KEK Proceedings 2000-20.

[9] Tsuji, S., Narihiro, N. and Oita, M. (2014) Absorbed Dose Conversion Factor in the Farmer Type Ionization Chamber. Proceedings of the 21st EGS Users' Meeting in Japan, KEK Proc. 2014-6, 25.

[10] Takeuchi, A., Raich, T., Yoshida, R., Kojima, K., Niwa, M., Komori, M. and Oguchi, H. (2013) Effect of the Difference in Electron Cutoff Energy on Surface Dose Calculation in the Monte Carlo Linear Accelerator Simulation of Megavoltage Photon Beams. Proceeding of the 20th EGS Users' Meeting in Japan, KEK Proc 2013-6, 30.

[11] Kondo, S., Haba, T., Hayashi, D., Numamoto, H., Ishii, T. and Koyama, S. (2012) Verification of Pin-Photo Diode Detector Characteristics Using EGS5. Proceeding of the 19th EGS Users' Meeting in Japan, KEK Proc 2012-7, 16.

[12] Kumagai, S., Okajima, M., Takaya, H., Arai, N., Waga, K., Tanaka, T., Yasuda, M., Takimoto, K., Kotoku, J. and Kobayashi, T. (2012) The Improvement of Discrepancy between Radiotherapy Treatment Planning System and Verification System Using Effective Density Method. Proceeding of the 19th EGS Users' Meeting in Japan, KEK Proc 2012-7, 66.

[13] Rachi, T., Iwamoto, Y., Tamura, M., Ota, K., Shimosato, T., Obata, Y. and Komori, M. (2012) Study of Collimator Scatter Factor (Sc) and Phantom Scatter Factor (Sp) Using Monte Carlo Simulation. Proceeding of the 19th EGS Users' Meeting in Japan, KEK Proc 2012-7, 76.

[14] Detectors for Relative and Absolute Dosimetry Ionisation Chambers and Diode Detectors. www.Iba-dosimetry.com

[15] Rogers, D.W.O., Walters, B.R.B. and Kawrakow, I. (2005) BEAMnrc User's Manual, NRC Report PIRS-509(rev I). 